\title{
CONCEPÇÕES DOS PROFESSORES UNIVERSITÁRIOS DE MATEMÁTICAS SOBRE A IMPLEMENTAÇÃO E USO DAS TICS PARA O ENSINO DE CONTEÚDOS MATEMÁTICOS.
}

\author{
CALI/Exterior MAIO/2018
}

\author{
Jakeline Amparo Villota Enríquez - USC - javillota@hotmail.com \\ Tipo: Investigação Científica (IC) \\ Natureza: Descrição de Projeto em Andamento \\ Categoria: Métodos e Tecnologias \\ Setor Educacional: EDUCAÇÃO SUPERIOR
}

\begin{abstract}
RESUMO
Dentro das concepções dos professores de matemática, podemos encontrar aqueles que têm a ver com o uso e a implementação das diferentes ferramentas tecnológicas, como celulares, tablets, computadores, entre outros, em sala de aula. Essas concepções estão ligadas ao processo de integração de novos artefatos tecnológicos durante o ensino e aprendizagem das matemáticas. Apesar das mudanças na prática pedagógica que estão ocorrendo ao introduzir a tecnologia nas salas de aula, os professores e suas concepções não estão sendo levados em conta. Por isso, é hora de analisar as concepções dos professores sobre a implementação e uso das TICs para o ensino de conteúdos matemáticos.
\end{abstract}

Palavras-chave: Concepções, tecnologias educativas, reforma curricular, prática pedagógica 
Introducción

Actualmente, en la investigación en Educación Matemática, un campo de gran interés es el relativo a las concepciones de los profesores. El interés por el estudio de las concepciones de los profesores radica en la influencia que ejercen en el proceso de enseñanza/aprendizaje de las matemáticas (Pajares, 1992; Defez, 2005; Bohorquez, 2014; De la Pienda, 1992; Moreno, 2000; Ponte, 1994; Furtado, 2014, Villota \& González, 2017). Por otro lado, esas concepciones pueden estar relacionadas con sus creencias. Por ello, algunos investigadores tienden a utilizar ambos términos como sinónimos, tal como lo argumentan Dodera et al. (n.d. p. 6):

"Thompson (1992) las diferencia explícitamente al expresar que las concepciones están compuestas de creencias y otras representaciones, pero en otros contextos las trata como sinónimos. Llinares (1991) reconoce que entre conocimiento, creencias y concepciones existen diferencias sutiles".

El estudio de esta temática se ha realizado tanto desde el punto de vista general (Hofer \& Pintrich, 1997; Koulaidis \& Ogborn, 1995; Porlan et al., 1998; Norton et al., 2005; Samuelowicz \& Bain, 1992; Hativa, 2000) como particular (Thompson, 1992; Gil \& Rico, 2003; Llinares, 1991; D’Amore \& Fandiño, 2004; Hudson, 1999) centrado en las matemáticas. Sin embargo, se han desarrollado pocas investigaciones de esta índole en el nivel universitario, por lo que su estudio resulta de gran interés.

A pesar de los cambios en la práctica pedagógica que se están produciendo al introducir la tecnología en las aulas, no se está teniendo en cuenta a los profesores y sus concepciones. Sin embargo, Goos \& Bennison (2002), reconocen que la tecnología juega un papel marginal en las aulas de matemáticas. Por ello es el momento de analizar las concepciones de los profesores en torno a la implementación y uso de las TICs para la enseñanza de contenidos matemáticos. Se pretende determinar cuáles son los diferentes elementos que integran estas concepciones, tales como la formación en el campo disciplinar (contenidos matemáticos), las experiencias (afectivas-emocionalesacadémicas), los componentes conductuales, entre otros.

El objetivo general de esta investigación es:

Determinar las concepciones de los profesores universitarios de matemáticas sobre la implementación y uso de las TICs para la enseñanza de contenidos matemáticos.

Para alcanzar este objetivo general se han establecido los siguientes objetivos específicos: 
1. Describir y caracterizar las concepciones que tienen los profesores universitarios de Matemáticas sobre la implementación y uso de las TICs.

2. Develar la influencia de la trayectoria profesional y la formación académica de los profesores universitarios de Matemáticas en la construcción de las concepciones sobre la implementación y uso de las TICs para la enseñanza de contenidos matemáticos.

3. Relacionar las concepciones de los profesores universitarios de matemáticas sobre la implementación de las TICs con la práctica pedagógica y las políticas educativas.

4. Determinar si el proceso de alfabetización integral sobre la implementación de las TICs influye en el proceso de construcción y/o cambio de las concepciones de los profesores universitarios de matemáticas.

5. Comparar las concepciones de los profesores universitarios de matemáticas que implementan las TICs y aquellos que aún no lo realizan en sus clases presenciales desde la práctica pedagógica, la trayectoria profesional y campo disciplinar

Metodología de la investigación

El análisis de datos obtenidos del cuestionario se realizará utilizando el análisis de contenido ("el análisis de contenido puede y debe ser utilizado en educación, pero dentro de parcelas específicas de estudio," López (2002, p. 168)). Las entrevistas se grabarán (en audio/vídeo) y posteriormente se harán las transcripciones correspondientes. Se categorizarán los datos obtenidos y se establecerán los códigos adecuados tal como señala Charmaz (2009). Esto implicará realizar una segmentación adecuada de las transcripciones (Cohen et al., 2007) que posteriormente se podrá resumir para obtener los resultados.

Los participantes en este estudio serán profesores universitarios de matemáticas, que imparten materias disciplinares como, por ejemplo: matemáticas fundamentales, Cálculo I, Cálculo II, entre otros; así como cursos relacionado con la Didáctica y la Historia de las Matemáticas entre los que tenemos: Didáctica de la Aritmética, Didáctica de la Geometría, Historia de las Matemáticas, etc. En total serán doce los profesores universitarios de matemáticas los que participarán de este estudio.

La selección de los profesores universitarios de matemáticas se hará atendiendo a criterios de afinidad con las asignaturas antes mencionadas; es decir, son profesores que enseñan matemáticas y disciplinas relacionadas con la Educación Matemática. Además, los profesores universitarios de matemáticas deben manifestar abierta y responsablemente su participación con el propósito de que a futuro no se convierta en una limitación para el desarrollo de esta investigación. 
La coherencia sobre los instrumentos de recolección de la información se dará en la medida de que los ítems tanto del cuestionario como de las entrevistas estén ligados con el objetivo a alcanzar; es decir, la información a recoger debe estar relacionada con el objetivo que se desea obtener. En cuanto a la consistencia de los instrumentos de recolección de información esta permitirá analizar el objetivo propuestos a partir de las informaciones recogidas, brindando una solides entre los métodos a implementar para el análisis de la información y la información recolectada. recogida.

\section{Referencias}

Bohorquez, L. (2014). Las creencias vs las concepciones de los profesores de matemáticas y sus cambios. ISBN: 978-84-7666-210-6. Artículo 1611. Memorias del Congreso Iberoamericano de Ciencia, Tecnología, Innovación y Educación. Buenos Aires, Argentina.

Contreras, L. (1998). Resolución de problemas. Un análisis exploratorio de las concepciones de los profesores acerca de su papel en el aula. Tesis doctoral Huelva: Universidad de Huelva.

Contreras, P. S. (2010). Las creencias curriculares de los profesores de ciencias: una aproximación a las teorías implícitas sobre el aprendizaje. Horizontes Educacionales. v. 15, n. 1. pp. 23-36.

Cohen, L.; Manion, L.; Morrison, K. (2007) Research methods education. (6th ed.). London: Routledge.

Charmaz, K. A. (2009). Construção da teoria fundamentada: guia prático para análise qualitativa. Tradução Joice Elias Costa. Porto Alegre: Artmed.

De la Pienda, J. A. (1999). Filosofía de las creencias. Revista de Filosofía de la Universidad de Costa Rica, 92, 239-48.

Defez, A. (2005). ¿Qué es una creencia? Logos: Anales del Seminario de Metafísica. 38(38), 199-221.

D’Amore, B. \& Fandiño Pinilla M. I. (2004). Cambios de convicciones en futuros profesores de matemática de la escuela secundaria superior. Epsilon. 20 (1), 25 - 43.

Dodera, M. G. (n. d.) Concepciones y creencias de profesores sobre enseñanza y aprendizaje de la matemática. Buenos Aires, Argentina. Recuperado en: http://www.soarem.org.ar/Documentos/39\%20Dodera.pdf . Acceso: 01 de noviembre de 2017.

Furtado, M. R. (2014). Uma Discussão Acerca do Conceito de Crença (Teses de mestrado). Universidade de Lisboa.

García, M. (1997). Análisis del conocimiento profesional del profesor de matemáticas de enseñanza secundaria y el concepto de función como objeto de enseñanza-aprendizaje. Aportaciones metodológicas. Tesis doctoral Sevilla: Universidad de Sevilla. 
García, L., Azcárate, C., \& Moreno, M. (2006). Creencias, concepciones y conocimiento profesional de profesores que enseñan cálculo diferencial a estudiantes de ciencias económicas. Revista Latinoamericana de Investigación en Matemática Educativa [RELIME], 9(1), 85-116.

Gil Cuadra, F. y Rico Romero, L. (2003). Concepciones y creencias del profesorado sobre enseñanza y aprendizaje de las matemáticas. Enseñanza de las ciencias, 21(1), 27-47.

Goos, M., \& Bennison, A. (2002). Building learning communities to support beginning teachers' use of technology. Annual Conference of the Australian Association for Research in Education. Retrieved December 14, 2008, from http://www.aare.edu.au/02pap/goo02058. htm

Goetz, J.P.y Lecompte, M.D. (1988). Etnografía y diseño cualitativo en investigación educativa. Madrid: Ed Morata.

Handal, B., \& Herrington, T. (2003). Mathematics teachers' beliefs and curriculum reform. Mathematics Education Research Journal.15(1), 59-69

Hativa, N. (2000). Teacher thinking, belief, and knowledge in higher education: an introduction. Instructional Science, 28(5) 331-334.

Hofer, B. K. y Pintrich, P. R. (1997). The development of epistemological theories: Beliefs about knowledge and knowing and their relation to learning. Review of Educational Research, 67(1), 88-140. doi: 10.3102/00346543067001088.

Hudson et al. (1999). Didaktik/fachdidaktik as science(-s) of the teaching profession?. Thematic Network of Teacher Education Europe 2(1) p. 1-261. ISSN 1403-5782.

Houston, R. (ed.) (1990): Handbook of Research on Teacher Education. Nueva York: McMillan

Koulaidis, Vasilis y Jon Ogborn (1995), Science teachers' philosophical assumptions: how well do we understand them? International Journal of Science Education, 17(3), 273-283.

Llinares S. (1991). La formación de profesores de matemáticas. Sevilla: GID.

Martínez, M. S. (2003). Concepciones sobre la enseñanza de la resta: un estudio en el ámbito de la formación permanente del profesorado. Tesis de doctorado. Departamento de Didáctica de la Matemática y las ciencias experimentales. Universidad Autónoma de Barcelona.

Moreno, M. (2000). El profesor universitario de matemáticas: estudio de las concepciones y creencias acerca de la enseñanza de las ecuaciones diferenciales (Tesis doctoral). Universidad Autónoma de Barcelona.

Norton, L. Richardson, J. y Hartley, J. (2005), Teachers' beliefs and intentions concerning teaching in higher education. Higher Education, 50(4), 537-571

Pajares, M. F. (1992). Teachers' beliefs and educational research: Cleaning up a messy 
construct. Review of Educational Research, 62(3), 307-332.

Ponte, J. P. (1994). Mathematics teacher's professional knowledge. En J. P. Ponte y J.

F. Matos (Eds.), Proceedings PME XVIII (vol 1, pp. 195 -210). Lisboa, Portugal.

Porlán, R., Rivero, A. Martín, R. (1998), Conocimiento profesional y epistemología de los profesores, II: estudios empíricos y conclusiones. Enseñanza de las Ciencias, 16(2), 271-288.

Samuelowicz, K. y Bain, J. (1992), Conceptions of teaching held by academic teachers. Higher Education, 24, 93-111.

Thompson, A. (1992). Teacher's beliefs and conceptions: A synthesis of research. En D.

A. Grouws (Ed.), Handbook of research on mathematics teaching and learning (pp. 127-146). Nueva York: Macmillan. 